\title{
Sequencing and iterative assembly of Ixiolirion tataricum plastome from total DNA using 2nd and 3rd generation HTS platforms
}

\author{
Omelchenko D.O. ${ }^{1,2 *}$, Krinitsina A.A. ${ }^{2}$, Logacheva M.D. ${ }^{1,2,3}$, Antipin M.I. ${ }^{2}$, \\ Speranskaya A.S. ${ }^{2,4}$ \\ ${ }^{1}$ Institute for Information Transmission Problems, RAS, Moscow, Russia \\ ${ }^{2}$ Lomonosov Moscow State University, Moscow, Russia \\ ${ }^{3}$ Skolkovo Institute of Science and Technology, Moscow, Russia \\ ${ }^{4}$ Central Research Institute of Epidemiology, Moscow, Russia \\ *e-mail: omdeno@gmail.com
}

The Ixiolirion genus is a systematically isolated group, the exact position of which within the order Asparagales is not completely clear. Chloroplast genomes are commonly $\sim 150 \mathrm{~kb}$ long and widely used for phylogenetic reconstruction. We've collected the tiny specimen of I. tataricum in the spurs of Kyrgyz Alatoo ridge, Republic of Kyrgyzstan in 2018 with the aim of plastome sequencing. The amount and condition of sample was not sufficient for laboratory manipulations to extract chloroplast fraction, and we couldn't repeat the sampling. Therefore, we had to extract total DNA and perform WGS using Illumina MiSeq. The total numbers of reads were theoretically enough for complete plastome assembling, but in fact only up to $\sim 25 \mathrm{~kb}$ long contigs were assembling (using whole library with Spades 3.13.0 assembler). To improve assembling we've performed additional sequencing by Ion Torrent and Oxford Nanopore HTS platforms. We have tested different approaches to extract chloroplast reads and assemble. The best approach we've found includes filtering by k-mer frequency and combined iterative filtering and assembly with enrichment of chloroplast reads and depletion of nuclear and bacterial contamination reads by separation of chloroplast and non-chloroplast nodes and clusters in de Bruijn graphs of Spades' assemblies using Bandage software, Blastn and BBmap. Assembly has much improved containing longer contigs and covering most of the LSC, SSC and IRs regions. We have found 5,527 bp inversion in SSC region, similar to the inversion we have observed in A. paradoxum; and that I. tataricum draft plastome sequence showed the highest similarity to complete plastome of Astelia pumila, compared to all other complete plastomes from GenBank.

Acknowledgements: This study was supported by the Russian Science Foundation. This work was supported by the RFBR grant number 18-04-01203. 